Supplement of Hydrol. Earth Syst. Sci., 21, 1137-1147, 2017

http://www.hydrol-earth-syst-sci.net/21/1137/2017/

doi:10.5194/hess-21-1137-2017-supplement

(C) Author(s) 2017. CC Attribution 3.0 License.

(c) (i)

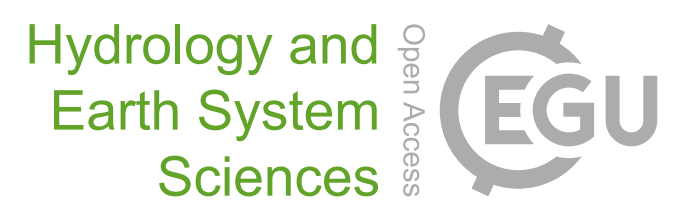

Supplement of

\title{
Developing a representative snow-monitoring network in a forested mountain watershed
}

Kelly E. Gleason et al.

Correspondence to: Kelly E. Gleason (kgleason@dri.edu)

The copyright of individual parts of the supplement might differ from the CC-BY 3.0 licence. 
Table S1. Accuracy assessment matrix comparing the BRT classes derived from the normal snow year 2009 with those from the high snow year 2008. Overall there is less error in the lowest and highest elevation BRT classes, whereas the mid- elevations there is more error between models. Many classes were reassigned when the BRT model was rerun between years, underestimating the accuracy of the overall spatial variability between models.

\begin{tabular}{|c|c|c|c|c|c|c|c|c|c|c|c|c|c|c|c|c|c|c|c|c|c|c|}
\hline \multicolumn{23}{|l|}{ BRT Class } \\
\hline 2009 & 1 & 2 & 3 & 4 & 5 & 6 & 7 & 8 & 9 & 10 & 11 & 12 & 13 & 14 & 15 & 16 & 17 & 18 & 19 & 20 & 21 & Comission \\
\hline 2008 & & & & & & & & & & & & & & & & & & & & & & error (\%) \\
\hline 1 & 55402 & 6035 & & & & & & & & & & & & & & & & & & & & 10 \\
\hline 2 & & 16467 & & & & & & & & & & & & & & & & & & & & 0 \\
\hline 3 & & 369 & 22960 & & & & & & & & & & & & & & & & & & & 2 \\
\hline 4 & & 52 & & 3930 & & & & & & & & & & & & & & & & & & 1 \\
\hline 5 & & & & & 9879 & & & & & & & & & & & & & & & & & 0 \\
\hline 6 & & & & & 5486 & & & & & & & & & & & & & & & & & 100 \\
\hline 7 & & & & & & 3232 & 3232 & & & & & & & & & & & & & & & 50 \\
\hline 8 & & & & & & & & 4667 & & & & & & & & & & & & & & 0 \\
\hline 9 & & & & & & & & & 2524 & & & & & & & & & & & & & 0 \\
\hline 10 & & & & & & & & 2053 & & 4007 & & & & & & & & & & & & 34 \\
\hline 11 & & & & & & & & & & 5276 & 5740 & & & & & & & & & & & 48 \\
\hline 12 & & & & & & & & & 486 & & & 2900 & & & & & & & & & & 14 \\
\hline 13 & & & & & & & & & & & 1965 & 339 & 5421 & & & & & & & & & 30 \\
\hline 14 & & & & & & & & & & & & & 5252 & 4338 & 617 & & & & & & & 57 \\
\hline 15 & & & & & & & & & & & & & & 13692 & 1948 & 719 & & & & & & 88 \\
\hline 16 & & & & & & & & & & & & & & & & 10260 & 14155 & & & & & 58 \\
\hline 17 & & & & & & & & & & & & & & & & & & 23580 & & & & 100 \\
\hline 18 & & & & & & & & & & & & & & & & & & & 5931 & 705 & & 100 \\
\hline 19 & & & & & & & & & & & & & & & & & & & & 1850 & & 100 \\
\hline 20 & & & & & & & & & & & & & & & & & & & & 1057 & 1025 & 51 \\
\hline 21 & & & & & & & & & & & & & & & & & & & & & 2039 & 0 \\
\hline Omission & 0 & 28 & 0 & 0 & 36 & 100 & 0 & 31 & 16 & 57 & 26 & 10 & 49 & 76 & 24 & 7 & 100 & 100 & 100 & 71 & 33 & \\
\hline error (\%) & & & & & & & & & & & & & & & & & & & Overal & all acc & curacy & 63 \\
\hline
\end{tabular}


Table S2. Accuracy assessment matrix comparing the BRT classes derived from the normal snow year 2005 with those from the high snow year 2008. Overall there is less error in the lowest and highest elevation BRT classes, whereas the mid- elevations there is more error between models. Many classes were reassigned when the BRT model was rerun between years, underestimating the accuracy of the overall spatial variability between models.

\begin{tabular}{|c|c|c|c|c|c|c|c|c|c|c|c|c|c|c|c|c|c|c|c|c|c|c|}
\hline \multicolumn{23}{|l|}{ BRT Class } \\
\hline 2009 & 1 & 2 & 3 & 4 & 5 & 6 & 7 & 8 & 9 & 10 & 11 & 12 & 13 & 14 & 15 & 16 & 17 & 18 & 19 & 20 & 21 & Comission \\
\hline 2005 & & & & & & & & & & & & & & & & & & & & & & error (\%) \\
\hline 1 & 55402 & 22923 & 22960 & 3930 & 15365 & 3232 & 6013 & 3365 & 2243 & & & & & & & & & & & & & 59 \\
\hline 2 & & & & & & & & 3355 & & 9283 & 5840 & & & & & & & & & & & 100 \\
\hline 3 & & & & & & & & & 767 & & & 2900 & & & & & & & & & & 100 \\
\hline 4 & & & & & & & & & & & 1965 & & 9212 & 12939 & & & & & & & & 100 \\
\hline 5 & & & & & & & & & & & & & & 5091 & 757 & 3973 & & & & & & 100 \\
\hline 6 & & & & & & & & & & & & 339 & 1461 & & 1808 & 879 & & & & & & 100 \\
\hline 7 & & & & & & & & & & & & & & & & 3718 & & & & & & 100 \\
\hline 8 & & & & & & & & & & & & & & & & & 2194 & & & & & 100 \\
\hline 9 & & & & & & & & & & & & & & & & & 3622 & & & & & 100 \\
\hline 10 & & & & & & & & & & & & & & & & & 2697 & & & & & 100 \\
\hline 11 & & & & & & & & & & & & & & & & & 3702 & & & & & 100 \\
\hline 12 & & & & & & & & & & & & & & & & & 1815 & & & & & 100 \\
\hline 13 & & & & & & & & & & & & & & & & & & 7239 & & & & 100 \\
\hline 14 & & & & & & & & & & & & & & & & & & 4776 & & & & 100 \\
\hline 15 & & & & & & & & & & & & & & & & & & 4045 & & & & 100 \\
\hline 16 & & & & & & & & & & & & & & & & & & 2347 & & & & 100 \\
\hline 17 & & & & & & & & & & & & & & & & & & 3253 & & & & 100 \\
\hline 18 & & & & & & & & & & & & & & & & & & 1923 & 512 & & & 21 \\
\hline 19 & & & & & & & & & & & & & & & & & & & 3857 & & & 0 \\
\hline 20 & & & & & & & & & & & & & & & & & & & 1562 & 3612 & 421 & 35 \\
\hline 21 & & & & & & & & & & & & & & & & & & & & & 2643 & 0 \\
\hline \multirow{2}{*}{$\begin{array}{l}\text { Omission } \\
\text { error (\%) }\end{array}$} & 0 & 100 & 100 & 100 & 100 & 100 & 100 & 100 & 100 & 100 & 100 & 100 & 100 & 100 & 100 & 100 & 100 & 92 & 35 & 0 & 14 & \\
\hline & & & & & & & & & & & & & & & & & & \multicolumn{4}{|c|}{ Overall accuracy } & 28 \\
\hline
\end{tabular}

